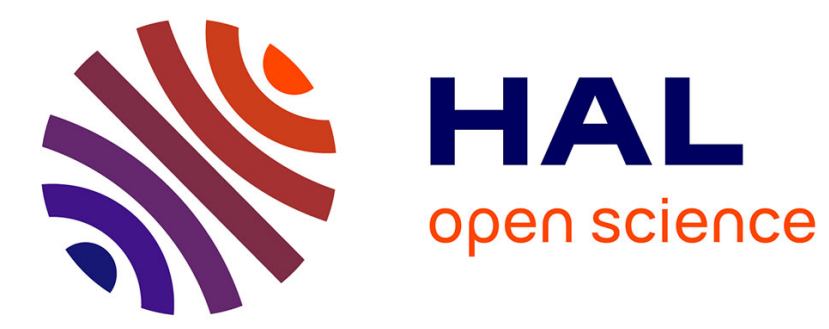

\title{
On Fairness via Picking Sequences in Allocation of Indivisible Goods
}

Laurent Gourvès, Julien Lesca, Anaëlle Wilczynski

\section{To cite this version:}

Laurent Gourvès, Julien Lesca, Anaëlle Wilczynski. On Fairness via Picking Sequences in Allocation of Indivisible Goods. 7th International Conference on Algorithmic Decision Theory (ADT-21), Nov 2021, Toulouse, France. pp.258-272, 10.1007/978-3-030-87756-9_17 . hal-03406848

\section{HAL Id: hal-03406848 \\ https://hal.archives-ouvertes.fr/hal-03406848}

Submitted on 18 Nov 2021

HAL is a multi-disciplinary open access archive for the deposit and dissemination of scientific research documents, whether they are published or not. The documents may come from teaching and research institutions in France or abroad, or from public or private research centers.
L'archive ouverte pluridisciplinaire HAL, est destinée au dépôt et à la diffusion de documents scientifiques de niveau recherche, publiés ou non, émanant des établissements d'enseignement et de recherche français ou étrangers, des laboratoires publics ou privés. 


\title{
On Fairness via Picking Sequences in Allocation of Indivisible Goods ${ }^{\star}$
}

\author{
Laurent Gourvès $^{1}$, Julien Lesca ${ }^{1}$, and Anaëlle Wilczynski ${ }^{2}$ \\ 1 Université Paris-Dauphine, Université PSL, CNRS, LAMSADE, 75016, Paris, \\ France, \{laurent.gourves, julien.lesca\}@dauphine.fr \\ 2 MICS, CentraleSupélec, Université Paris-Saclay, France \\ anaelle.wilczynski@centralesupelec.fr
}

\begin{abstract}
Among the fairness criteria for allocating indivisible resources to a group of agents, some are based on minimum utility levels. These levels can come from a specific allocation method, such as maximin fair-share criterion which is based on the cut-and-choose protocol. We propose to analyze criteria whose minimum utility levels are inspired by picking sequences, a well-established protocol for allocating indivisible resources. We study these criteria and investigate their connections with known fairness criteria, enriching the understanding of fair allocation of indivisible goods.
\end{abstract}

Keywords: Fair division - Resource allocation - Computational Social Choice.

\section{Introduction}

Fair division of indivisible goods is a fundamental and challenging question in collective decision making that has been widely investigated $[6,10,22]$. Many criteria have been proposed in the literature in order to evaluate the fairness of an allocation when agents express preferences over bundles of goods via additive utilities. A very natural criterion is envy-freeness $(E F)[18,30]$, a comparisonbased criterion which asks that no agent prefers the bundle assigned to another agent over her own assigned bundle of goods. This criterion notably requires that agents are aware of the other agents' allocation. Alternatively, many criteria simply impose, for an allocation to be considered fair, that each agent gets a utility for her assigned bundle that is greater than or equal to a predefined minimum utility level, called a fair guarantee [5]. As defined by Bogomolnaia et al. [5], a fair guarantee for an agent is a utility level defined only according to the utility function of the agent and the number of agents $n$. One can cite the proportionality (Prop) fair guarantee [28] where each agent must get at least a utility equal to her value for the whole set of goods divided by $n$. In addition, a fair guarantee can be defined according to a given allocation procedure like,

\footnotetext{
* This research benefited from the support of the FMJH Program PGMO under grant DAMPER and from the support of EDF, Thales, and Orange.
} 
e.g., the maximin share (MMS) [13] or the min-max-fair-share (mFS) [9] which are computed thanks to the cut-and-choose protocol. In this article, we define several fairness criteria whose fair guarantee can be computed thanks to picking sequences.

In the well-established allocation protocol of picking sequences $(P S)[7,12$, 20], all goods are initially available and, given a sequence of agents (a.k.a. policy), each agent picks at her turn an object among the remaining ones. Understanding which allocations emerge from such a mechanism has been done, for example, by Brams et al. [11], and Aziz et al. [4]. Moreover, picking sequences have been widely studied in a strategic perspective where agents may choose not to pick their best object $[2,8,19,29]$. Non strategic agents are said to be sincere.

One of the main assets of picking sequences is their simplicity: everyone can quickly understand how they work and they are easy to implement. Thus, they are good candidates for sharing resources. If the final allocation is not built with a picking sequence, then an agent may advocate for it and claim that her utility must be as good as the one resulting from a picking sequence that she has in mind. However, the number of possible policies is huge and, on top of this, every agent can have her own policy in mind. This offers a number of combinations which is undoubtedly too large. For a positive integer $p$ bounded by the number of agents, we propose a simple criterion named $\mathrm{PS}_{p}$ in which the fair guarantee of every agent is her utility for a subset of objects built as follows. Rank the objects from best to worst under the agent's preference, and keep the items whose ranks are multiples of $p$. An agent would be endowed such a set in a sincere picking sequence if her positions in the policy were multiples of $p$, and if the other agents had the same preference. Indeed, without knowing the others' preferences, an agent may suppose that, in the worst case, everyone has the same object ranking as hers. In $\mathrm{PS}_{p}$, the parameter $p$ makes it possible to move gradually between a very optimistic scenario where all the agents choose first $(p=1)$, and a more pessimistic one where they all choose last $(p=n)$. PS criteria only rely on a very simple sequential allocation protocol, which is commonly known (think about composition of sports teams at school). Moreover, agents only need to know their assigned bundle, the number of agents and their own preferences over goods. Therefore, these criteria are easy to understand and can be naturally expressed as requirements by an agent.

The fact that the agent appears recursively in the policy is inspired by round robin, a well known method for allocating resources [3, 26]. Round robin falls into the class of recursively balanced (RB) policies [4], where each sequence of agents can be divided into rounds during which all the agents pick an object exactly once (all rounds are identical in round robin). At any step of the sequence, the agents have chosen almost the same number of objects. Without any prior knowledge on the agents' utility functions, letting the agents pick the same number of times, leading then to an even-shares division, constitutes a natural first argument for equity [10]. Moreover, it is known from Aziz et al. [3] that picking sequences with $\mathrm{RB}$ policies generate allocations that are envy-free up to one good (EF1) [13, 23], a well-accepted fairness criterion which relaxes envy-freeness. 
The round robin share criterion introduced by Conitzer et al. [17] in the context of public decision making corresponds to $\mathrm{PS}_{n}$. The round robin share provides a one half approximation to Prop1, a relaxed version of proportionality. Conitzer et al. [17] focus on mechanisms that satisfy this criterion among others, whereas we focus on the properties of the PS criteria.

Contribution and organization. Section 2 contains a formal definition of the model, a review of classical fairness concepts, some common relaxations up to some goods, and a map explaining how all these notions relate. We notably complement the state of the art on the relations between relaxations of envy-freeness and proportionality. The PS criteria are introduced in Section 3. Analogously to many relaxed criteria based on the satisfaction of the fairness requirement up to the addition of some goods in the agent's bundle (like, e.g., EF1 for envy-freeness or Prop1 for proportionality), we also study relaxations of the PS criteria up to some goods. For a given allocation of goods, the satisfaction of a PS criterion can be checked in polynomial time. We identify in Section 4 the PS criteria for which a satisfying allocation always exists, and when it is not the case, we settle the complexity of deciding the existence of a satisfying allocation in a given instance. Contrary to many classical criteria, we identify two non-trivial PS criteria, namely $\mathrm{PS}_{n}$ and $\mathrm{PS}_{1} 1$ (the relaxation up to one good of $\mathrm{PS}_{1}$ ), for which a satisfying allocation always exists. Afterwards, we provide a complete picture of the implications that relate the PS criteria and the classical fairness concepts (Section 5), as well as their relaxations up to some goods. All these results are summarized in Figure 1. Finally, we complement our study with experiments which give an intuition on how well fairness criteria can be compatible with efficiency. Due to space limitation, some proofs are omitted.

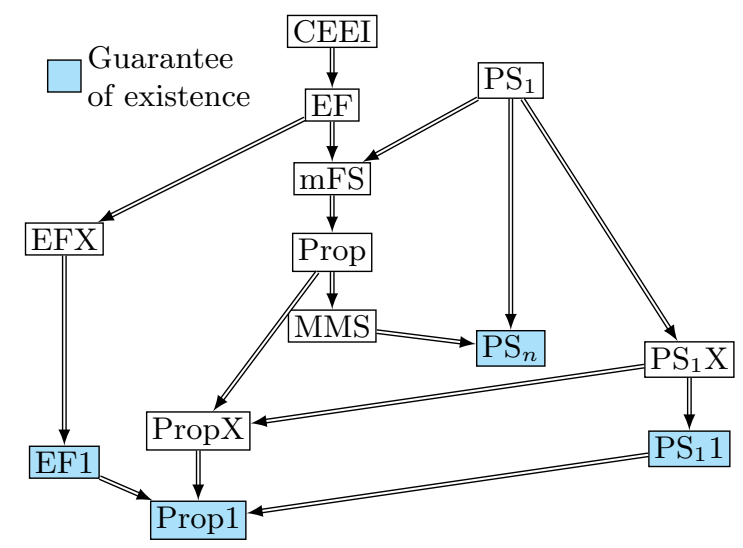

Fig. 1. Summary of the relations among fairness criteria and their existence guarantee (critX stands for the relaxation of the criterion crit up to any good). An arrow from criterion $A$ to criterion $B$ means that $A$ implies $B$ ( $A$ is stronger than $B)$. If there is no path from $A$ to $B$ then $A$ is not stronger than $B$. 


\section{Fair Division of Indivisible Goods}

\subsection{The setting}

We are given a set $N=\{1, \ldots, n\}$ of $n \geq 2$ agents and a set $M=\left\{x_{1}, \ldots, x_{m}\right\}$ of $m$ indivisible resources (or objects) which are goods. The agents have cardinal preferences over the bundles of objects, expressed via utility function $u_{i}: 2^{M} \rightarrow$ $\mathbb{R}^{+}$for each agent $i$. We assume that the utilities are additive, i.e., for each bundle of objects $O$ and each agent $i, u_{i}(O)=\sum_{x_{j} \in O} u_{i}\left(\left\{x_{j}\right\}\right)$. For the sake of simplicity, we denote $u_{i}(\{x\})$ by $u_{i}(x)$. We represent the preferences by an $(n \times m)$-matrix where the value in row $i$ and column $j$ corresponds to $u_{i}\left(x_{j}\right)$. Preferences are strict (on the objects) whenever $u_{i}(x) \neq u_{i}(y)$ for every agent $i$ and pair of objects $x$ and $y$. We denote by $o_{i}^{k}$ the $k^{\text {th }}$ most preferred object of agent $i$, for $1 \leq k \leq m$ (an arbitrary order over the objects is used in case of ties). We suppose, w.l.o.g., that the number of objects $m$ is a multiple of the number of agents $n$ (dummy objects with utility 0 can be added if it is not initially the case), and $q$ denotes the quotient $m / n$.

An allocation $\sigma$ is a mapping $\sigma: N \rightarrow 2^{M}$ such that $\sigma(i) \cap \sigma(j)=\emptyset$ for all agents $i$ and $j$, and $\bigcup_{i \in N} \sigma(i)=M$, where $\sigma(i)$ is the bundle assigned to agent $i$. $\mathcal{A}$ denotes the set of all allocations. The $n$-vector $u(\sigma)=\left(u_{1}(\sigma(1)), \ldots, u_{n}(\sigma(n))\right)$ describes the utilities that the agents obtain from allocation $\sigma$.

In this article, $[t]:=\{1, \ldots, t\}$ for all positive integers $t$.

\subsection{Classical fairness criteria}

For the sake of self-containedness, we recall some classical fairness criteria.

- Maximin share guarantee (MMS) [13]: Allocation $\sigma$ is MMS iff $u_{i}(\sigma(i)) \geq$ $m m s_{i}$ for every agent $i$, where $m m s_{i}=\max _{\sigma^{\prime} \in \mathcal{A}} \min _{j \in N} u_{i}\left(\sigma^{\prime}(j)\right)$.

- Proportionality (Prop) [28]: Allocation $\sigma$ is Prop iff $u_{i}(\sigma(i)) \geq \frac{1}{n} \sum_{x \in M} u_{i}(x)$, for every agent $i$.

- Min-max-fair-share guarantee (mFS) [9]: Allocation $\sigma$ is mFS iff $u_{i}(\sigma(i)) \geq$ $m f s_{i}:=\min _{\sigma^{\prime} \in \mathcal{A}} \max _{j \in N} u_{i}\left(\sigma^{\prime}(j)\right)$, for every agent $i$.

- Envy-freeness (EF) [18, 30]: Allocation $\sigma$ is EF iff $u_{i}(\sigma(i)) \geq u_{i}(\sigma(j))$ for all agents $i$ and $j$.

- Competitive Equilibrium with Equal Incomes (CEEI) (see, e.g., Moulin [24]): Allocation $\sigma$ is CEEI iff there exists a price vector $p \in[0,1]^{m}$ such that $\sigma(i) \in$ $\arg \max _{O \subseteq M}\left\{u_{i}(O): \sum_{o \in O} p_{o} \leq 1\right\}$ for every agent $i$.

An implication $A \Rightarrow B$ between criteria means that if an allocation satisfies $A$ for a given instance, then the same allocation also satisfies $B$ for the same instance. When such an implication holds, we say that criterion $A$ is stronger, or more demanding, than criterion $B$. All previous fairness criteria can be connected w.r.t. such implications to form the following "scale of fairness" [9].

$$
C E E I \Rightarrow E F \Rightarrow m F S \Rightarrow P r o p \Rightarrow M M S
$$

An allocation satisfying MMS is guaranteed to exist for two agents [9]. However, starting from 3 agents, there may not exist an MMS allocation [21, 27]. 
Common relaxations of envy-freeness and proportionality are based on satisfying the criterion up to a fixed number $c$ of goods, for $c$ a positive integer. Allocation $\sigma$ is proportional up to $c$ goods (Propc) [16] iff for every agent $i$, there exists $X^{i} \subseteq M \backslash \sigma(i)$ such that $\left|X^{i}\right| \leq c$ and $u_{i}(\sigma(i))+u_{i}\left(X^{i}\right) \geq u_{i}(M) / n$. Allocation $\sigma$ is envy-free up to $c$ goods (EFc) iff for all agents $i$ and $j$, there exists $X^{i} \subseteq \sigma(j)$ such that $\left|X^{i}\right| \leq c$ and $u_{i}(\sigma(i))+u_{i}\left(X^{i}\right) \geq u_{i}(\sigma(j))$. By definition, Prop $\Rightarrow \operatorname{Prop} c$ (resp., EF $\Rightarrow \mathrm{EF} c$ ) holds for all $c$, and Prop $c \Rightarrow \operatorname{Prop} c^{\prime}$ (resp., $\mathrm{EF} c \Rightarrow \mathrm{EF}^{\prime}$ ) whenever $c \leq c^{\prime}$. In addition, we show that $\mathrm{EF} c \Rightarrow \operatorname{Prop} c$ holds for all $c$.

Proposition 1. EFc $\Rightarrow$ Propc.

It follows that $\mathrm{EF} c \Rightarrow \operatorname{Prop} c^{\prime}$ whenever $c \leq c^{\prime}$. The existence of an allocation satisfying EF1 [13, 23] or Prop1 [17] i.e., $c=1$, is guaranteed for every instance. Relaxations up to one good have been strengthened to any good. Allocation $\sigma$ is proportional up to any good (PropX) [25] iff for every agent $i, u_{i}(\sigma(i))+$ $u_{i}(x) \geq u_{i}(M) / n$ holds for all $x \in M \backslash \sigma(i)$. By definition we have Prop $\Rightarrow$ PropX $\Rightarrow$ Prop1. Allocation $\sigma$ is envy-free up to any good (EFX) [15, 23] iff $u_{i}(\sigma(i))+u_{i}(x) \geq u_{i}(\sigma(j))$ for every pair of agents $i$ and $j$ and any object $x \in \sigma(j)$. It holds that $\mathrm{EF} \Rightarrow \mathrm{EFX} \Rightarrow \mathrm{EF} 1$.

Though EFc implies Prop $c$, we show that EFX does not imply PropX when $n>2$. Up to our best knowledge, this fact has not been explicitly stated before.

Proposition 2. If $n=2$ then $E F X \Rightarrow \operatorname{Prop} X$, but $E F X \nRightarrow \operatorname{Prop} X$ when $n>2$.

Proof. Suppose there are two agents. Take an instance and an EFX allocation $\sigma$. Take the viewpoint of an agent, say agent 1 . We have $u_{1}(\sigma(1))+u_{1}(x) \geq u_{1}(\sigma(2))$ for all $x \in \sigma(2)$. Add $u_{1}(\sigma(1))+u_{1}(x) \geq u_{1}(\sigma(2))$ to $u_{1}(\sigma(1))+u_{1}(x) \geq u_{1}(\sigma(1))$ in order to get that $2\left(u_{1}(\sigma(1))+u_{1}(x)\right) \geq u_{1}(\sigma(1))+u_{1}(\sigma(2))$. By definition, we also have $u_{1}(\sigma(1))+u_{1}(\sigma(2))=u(M)$. Therefore $\sigma$ satisfies PropX.

Suppose there are 3 agents and 5 objects $\left\{x_{1}, \ldots, x_{5}\right\}$ valued $(1,1,1,0.25,0.05)$ by agent 1 . The utility of agents 2 and 3 is 1 for all objects. Agents 1,2 and 3 get $\left\{x_{1}\right\},\left\{x_{2}, x_{3}\right\}$, and $\left\{x_{4}, x_{5}\right\}$, respectively. This allocation is EFX, but not PropX because $1.05=u_{1}\left(x_{1}\right)+u_{1}\left(x_{5}\right) \geq u_{1}(M) / 3=1.1$. We can extend this instance to any number of agents $n>3$.

\section{Picking Sequence (PS) Fairness Criteria}

We present new fairness criteria inspired by some picking sequences. A policy $\pi:\{1, \ldots, m\} \rightarrow N$ is a sequence of agents of size $m$, denoted by $\pi=\langle\pi(1), \ldots$, $\pi(m)\rangle$. A picking sequence is a sequential protocol asking agent $\pi(t)$ to pick an object within the set of remaining objects at stage $t$. A policy $\pi$ is recursively balanced (RB) [4] if $\pi$ can be decomposed into $q=\frac{m}{n}$ rounds, and each agent chooses an object exactly once at each round. Round robin is a special RB policy where all rounds are identical [3].

The PS fairness criteria use fair guarantees [5]. For every $p \in[n], \mathrm{PS}_{p}$ imposes that the utility of an agent $i$ for her share is at least $p s_{p}(i)$ where 


$$
p s_{p}(i):=\sum_{k=1}^{q} u_{i}\left(o_{i}^{(k-1) n+p}\right) .
$$

For example, agent $i$ would get utility $p s_{p}(i)$ in a sincere ${ }^{3}$ picking sequence if her turns in $\pi$ were all the multiples of $p$, and if the other agents had identical preferences. Without knowing the preferences of the others, agent $i$ considers the worst case where all the other agents have the same induced ordinal preferences as hers. In such a case, at each turn $k$, agent $i$ can only get her $((k-1) n+p)^{\text {th }}$ most preferred available object, i.e., $o_{i}^{(k-1) n+p}$.

Let $p s_{p}$ be the $n$-vector $\left(p s_{p}(1), \ldots, p s_{p}(n)\right)$. An allocation satisfies a PS criterion if it fulfills the PS fair guarantees for every agent and some common position $p$. It is important to note that allocations satisfying a PS criterion do not need to be generated by a picking sequence.

Definition 1 ( $\mathbf{P S}_{p}$ allocation). An allocation $\sigma \in \mathcal{A}$ is $P S_{p}$ if for every agent $i \in N, u_{i}(\sigma(i)) \geq p s_{p}(i)$.

By definition, $\mathrm{PS}_{p} \Rightarrow \mathrm{PS}_{p^{\prime}}$ holds for every $1 \leq p \leq p^{\prime} \leq n$. In this article, we pay particular attention to positions $p=1$ and $p=n$, which correspond to an optimistic and pessimistic view, respectively.

The PS fair guarantees are computable in polynomial time, by definition. Therefore, checking whether a given allocation satisfies a PS criterion is computationally easy. Whereas this polynomial-time verification also holds for proportionality and envy-freeness, this is not the case for CEEI, nor for MMS and mFS [9], although the two latter notions are also based on fair guarantees. Note that, contrary to envy-based criteria, the verification of satisfaction of a PS criterion does not even need to have access to other agents' allocation.

Like EF and Prop, $\mathrm{PS}_{p}$ can be relaxed up to some goods. Allocation $\sigma$ satisfies $\mathrm{PS}_{p}$ up to $c$ goods $\left(\mathrm{PS}_{p} c\right)$ iff for every agent $i$, there exists $X^{i} \subseteq M \backslash \sigma(i)$ such that $\left|X^{i}\right| \leq c$ and $u_{i}(\sigma(i))+u_{i}\left(X^{i}\right) \geq p s_{p}(i)$. By definition we have $\mathrm{PS}_{p} c \Rightarrow$ $\mathrm{PS}_{p^{\prime}} c \Rightarrow \mathrm{PS}_{p^{\prime}} c^{\prime}$ whenever $p \leq p^{\prime}$ and $c \leq c^{\prime}$. However, a $\mathrm{PS}_{p}$ allocation may not satisfy $\mathrm{PS}_{p-1} 1$, as stated below.

Proposition 3. $P S_{2}$ does not imply $P S_{1} 1$.

Proof. Consider an instance where $n=2$ and $m=6$. The utilities are:

$$
\left(\begin{array}{llllll}
24 & 16 & 12 & 6 & 5 & 2 \\
10 & 5 & 4 & 3 & 2 & 1
\end{array}\right)
$$

Allocation $\sigma$ (circles) is $\mathrm{PS}_{2}$ since $p s_{2}=(24,9)$ and $u(\sigma)=(24,15)$. However, it is not $\mathrm{PS}_{1} 1$ because $u_{1}(\sigma(1))+\max _{x \notin \sigma(1)} u_{1}(x)=24+16<41=p s_{1}(1)$.

Allocation $\sigma$ satisfies $\mathrm{PS}_{p}$ up to any good $\left(\mathrm{PS}_{p} \mathrm{X}\right)$ iff for every agent $i$, $u_{i}(\sigma(i))+u_{i}(x) \geq p s_{p}(i)$ holds for all $x \in M \backslash \sigma(i)$. It holds that $\mathrm{PS}_{p} \Rightarrow$ $\mathrm{PS}_{p} \mathrm{X} \Rightarrow \mathrm{PS}_{p} 1$. However, no relaxation up to any good implies a PS criterion with no relaxation, as stated below.

Proposition 4. $P S_{1} X$ does not imply $P S_{n}$.

\footnotetext{
${ }^{3}$ Agents always pick their favorite object.
} 


\section{Allocations Satisfying PS Criteria}

Observe first that a $\mathrm{PS}_{p}$ allocation may not exist if $p<n$ : Consider an instance where $n=m$ with agents having the same induced preference order and no object with zero utility. Every agent should receive one object but no agent wants the common least preferred object. However, when $p=n$, the existence is guaranteed for every number of goods $m$ because every allocation resulting from a picking sequence with an RB policy is $\mathrm{PS}_{n}$.

Proposition 5. Every allocation resulting from a sincere picking sequence with an $R B$ policy is $P S_{n}$.

Proof. Consider an allocation $\sigma$ resulting from a sincere picking sequence with an RB policy $\pi$, and take an arbitrary agent $i$. For each round $k$ of $\pi$, let $p_{i}(k)$ denote the position occupied by agent $i$ in $\pi$ during round $k$, while $x_{k}$ is the object picked by agent $i$ in round $k$. By definition, we have $u_{i}(\sigma(i))=\sum_{1 \leq k \leq q} u_{i}\left(x_{k}\right)$. Since agent $i$ is sincere and $p_{i}(k)-1$ objects have been taken before agent $i$ picks at round $k$, it follows that $u_{i}\left(x_{k}\right) \geq u_{i}\left(o_{i}^{p_{i}(k)}\right)$ for every round $k$. Thus, $u_{i}(\sigma(i)) \geq$ $\sum_{1 \leq k \leq q} u_{i}\left(o_{i}^{p_{i}(k)}\right)$. By definition of an RB sequence, $(k-1) n+1 \leq p_{i}(k) \leq k n$ holds. Therefore, we get that $u_{i}(\sigma(i)) \geq \sum_{1 \leq k \leq q} u_{i}\left(o_{i}^{k n}\right)=p s_{n}(i)$.

The converse of Proposition 5 is not true. That is, not every $\mathrm{PS}_{n}$ allocation can result from a picking sequence with an RB policy, as shown in the next example. This notably shows that allocations satisfying the PS criteria do not necessarily emerge from a picking sequence (in particular, agents do not necessarily get the same number of objects).

Example 1. Consider an instance where $n=2$ and $m=4$. The utilities are:

$$
\left(\begin{array}{llll}
20 & 3 & 2 & 1 \\
5 & 4 & 3 & 2
\end{array}\right)
$$

The encircled allocation $\sigma$ is $\operatorname{PS}_{n}$ since $p s_{n}=(4,6)$ and $u(\sigma)=(20,9)$. However, this allocation cannot result from a picking sequence with an RB policy since the two agents do not have the same number of objects.

Nevertheless, checking the existence of a $\mathrm{PS}_{p}$ allocation is hard for every constant $p<n$, even when $m=2 n$.

Theorem 1. Determining whether a $P S_{p}$ allocation exists is NP-complete, even when $m=2 n$ and $p<n$ is a constant.

Moreover, checking the existence of a $\mathrm{PS}_{1}$ allocation is hard even when $n=2$, showing that even checking the existence of a $\mathrm{PS}_{n-1}$ allocation is hard.

Theorem 2. Determining whether a $P S_{1}$ allocation exists is NP-complete, even when $n=2$.

However, an allocation satisfying the relaxation up to one good of $\mathrm{PS}_{1}$ always exists. 
Proposition 6. Every allocation resulting from a sincere picking sequence with an $R B$ policy is $P S_{1} 1$.

Proof. Consider the allocation $\sigma$ built with the sincere picking sequence that uses an RB policy. Take an agent $i$. Her objects are $\left\{o_{i}^{f(1)}, o_{i}^{f(2)}, \ldots, o_{i}^{f(q)}\right\}$ for some increasing function $f:[q] \rightarrow[m]$ where $f(j)$ is the rank in the preference order of $i$ of the object picked by $i$ during round $j$. Let $r$ be the smallest index such that $o_{i}^{r} \notin \sigma(i)$. Agent $i$ has in her share every object $o_{i}^{j}$ with $j<r$. Thus, $o_{i}^{f(j)}=o_{i}^{j}$ for all $j<r$. We deduce that $\sum_{j<r} u_{i}\left(o_{i}^{f(j)}\right)=$ $\sum_{j<r} u_{i}\left(o_{i}^{j}\right) \geq \sum_{j<r} u_{i}\left(o_{i}^{1+(j-1) n}\right) \quad$ (2). The policy being RB, we also have $u_{i}\left(o_{i}^{f(j)}\right) \geq u_{i}\left(o_{i}^{j n}\right) \geq u_{i}\left(o_{i}^{1+j n}\right)$ for all $j \in[q-1]$, from which we deduce that $\sum_{j=r}^{q-1} u_{i}\left(o_{i}^{f(j)}\right) \geq \sum_{j=r}^{q-1} u_{i}\left(o_{i}^{1+j n}\right)=\sum_{j=r+1}^{q} u_{i}\left(o_{i}^{1+(j-1) n}\right) \quad$ (3). Combine (2) and (3) with $u_{i}\left(o_{i}^{r}\right) \geq u_{i}\left(o_{i}^{1+(r-1) n}\right)$ to get that $u_{i}(\sigma(i))+u_{i}\left(o_{i}^{r}\right) \geq$ $\sum_{k=1}^{q} u_{i}\left(o_{i}^{1+(k-1) n}\right)=p s_{1}(i)$. In other words, $\sigma$ is $\mathrm{PS}_{1} 1$ for agent $i$.

Propositions 5 and 6, together with Proposition 1 from Aziz et al. [3], imply that a sincere picking sequence with an RB policy produces an allocation that simultaneously satisfies EF1, Prop1, $\mathrm{PS}_{1} 1$ and $\mathrm{PS}_{n}$.

By Proposition 6, a $\mathrm{PS}_{p} 1$ allocation exists for every $p \in[n]$. It is not the case for $\mathrm{PS}_{p} \mathrm{X}$, even when $p=n-1$ for any number $n$ of agents: Consider an instance where $m=2 n$ with the following preferences for every agent $i: u_{i}\left(x_{j}\right)=1$ for every $j \in[n-1], u_{i}\left(x_{j}\right)=1 / n$ for every $n \leq j \leq 2 n-1$ and $u_{i}\left(x_{2 n}\right)=0$. We have $p s_{n-1}(i)=1+1 / n$ for every agent $i$. To satisfy $\operatorname{PS}_{n-1} \mathrm{X}$, each agent $i$ must be in one of the following situations: $u_{i}(\sigma(i)) \geq 1+1 / n$, or $\sigma(i)=\left\{x_{k}, x_{2 n}\right\}$ for some $k \in[n-1]$, or $\sigma(i)=\left\{x_{j}: n \leq j \leq 2 n\right\}$. Making $n$ disjoint bundles under such conditions is impossible.

Since a $\mathrm{PS}_{1} 1$ allocation always exists, there is no need to consider relaxations up to $c$ goods for $p>1$ and $c>1$. Combined with the fact that the existence of $\mathrm{PS}_{p} \mathrm{X}$ allocations is not guaranteed even for $p=n-1$, we can focus on stronger relaxations and only consider criteria $\mathrm{PS}_{1} 1$ and $\mathrm{PS}_{1} \mathrm{X}$.

\section{$5 \quad$ Relations between Fairness Criteria}

We compare in this section the PS criteria with the classical fairness criteria of the literature given in Section 2.2. We will show that the ordered scale of fairness (1), completed with known relaxations of envy-freeness and proportionality, can be connected with the PS criteria as shown in Figure 1.

Surprisingly, the strongest requirement CEEI in the fairness scale (1) does not even imply $\mathrm{PS}_{n-1}$ and $\mathrm{PS}_{1} 1$ which are among the least demanding PS criteria.

Proposition 7. $C E E I \nRightarrow P S_{n-1}$ for any number of agents $n$ and $C E E I \nRightarrow P S_{1} 1$.

Proof. Consider an instance where $m=2 n$, and two integers $\alpha$ and $\beta$ such that $\beta n>\alpha>\beta(n-1)$ and $\beta>1$. The utilities are such that $u_{i}\left(x_{i}\right)=\alpha$ and $u_{i}\left(x_{j}\right)=0$ for every index $j \neq i$ and every agent $i \in\{1, \ldots, n-2\}$, and 
$u_{n-1}\left(x_{n-1}\right)=u_{n-1}\left(x_{2 n}\right)=\alpha$ and $u_{n-1}(x)=0$ for every object $x \in M \backslash$ $\left\{x_{n-1}, x_{2 n}\right\}$. The utility function of agent $n$ is such that $u_{n}\left(x_{j}\right)=\alpha$ for every index $j \in\{1, \ldots, n-1\}, u_{n}\left(x_{j}\right)=\beta$ for every index $j \in\{n, \ldots, 2 n-1\}$, and $u_{n}\left(x_{2 n}\right)=0$. Let us denote by $\sigma$ the allocation assigning object $x_{i}$ to every agent $i \in\{1, \ldots, n-2\}$, the bundle of objects $\left\{x_{n-1}, x_{2 n}\right\}$ to agent $n-1$ and the bundle $\left\{x_{n}, x_{n+1}, \ldots, x_{2 n-1}\right\}$ to agent $n$. Observe that allocation $\sigma$ is CEEI w.r.t. price vector $p$ given by $p_{i}=1$ for every $i \in\{1, \ldots, n-2\}, p_{n-1}=\frac{n}{n+1}, p_{i}=\frac{1}{n}$ for every $i \in\{n, n+1, \ldots, 2 n-1\}$ and $p_{2 n}=\frac{1}{n+1}$. However, allocation $\sigma$ is not $\mathrm{PS}_{n-1}$ because $u_{n}(\sigma(n))=\beta n<\alpha+\beta=u_{n}\left(o_{n}^{n-1}\right)+u_{n}\left(o_{n}^{2 n-1}\right)=p s_{n-1}(n)$, and thus is not $\mathrm{PS}_{p}$ for any position $p \in\{1, \ldots, n-1\}$. For $\mathrm{PS}_{1} 1$, it suffices to remark that the encircled allocation in the proof of Proposition 3 is CEEI w.r.t. price vector $p=(1,0.75,0.6,0.2,0.2,0.25)$ but not $\mathrm{PS}_{1} 1$.

It follows that none of the criteria of the scale of fairness implies $\mathrm{PS}_{p}$ when $p<n$, meaning that $\mathrm{PS}_{p}$ is not always "weaker" than any criterion of the scale of fairness. However, all criteria of the scale of fairness imply the $\mathrm{PS}_{n}$ criterion.

Proposition 8. $M M S \Rightarrow P S_{n}$.

Proof. We prove that $m m s_{i} \geq p s_{n}(i)$ for every agent $i$. Take an allocation where the $\ell^{\text {th }}$ bundle $(1 \leq \ell \leq n)$ gathers all the $(\ell+k n)^{\text {th }}$ most preferred objects of agent $i$ for $0 \leq k<q$. The $n^{\text {th }}$ bundle, whose value is $p s_{n}(i)$, is the least preferred. Thus, $m m s_{i} \geq p s_{n}(i)$ because $m m s_{i}$ is agent $i$ 's maximum value for the worst bundle for every possible allocation.

Conversely, for $p>1$, the PS criteria are not stronger than any classical criterion either. Indeed, $\mathrm{PS}_{2}$ does not imply MMS, the least demanding criterion in the fairness scale (1) nor Prop1, thus no PS criterion with $p>1$ does.

Proposition 9. 1. $P S_{2} \nRightarrow M M S$ for any $n$,

2. $P S_{2} \nRightarrow$ Prop1,

3. $P S_{2} \nRightarrow$ Propc for large enough $m$ and any $c$.

Sketch of proof. We only present case (2) here. Consider an instance where $n=3$ and $m=6$. The utilities are:

$$
\left(\begin{array}{cccccc}
24 & 16 & 15 & 14 & 8 & 7 \\
1 & 2 & 3 & 4 & 5 & 6 \\
2 & 4 & 6 & 8 & 10 & 12
\end{array}\right)
$$

The encircled allocation $\sigma$ is $\mathrm{PS}_{2}$ since $p s_{2}=(24,7,14)$. However, $\sigma$ is not Prop1 because $u_{1}(\sigma(1))+\max _{x \notin \sigma(1)} u_{1}(x)=24+16<42=u_{1}(M) / 2$.

However, $\mathrm{PS}_{1}$ implies the mFS criterion.

Proposition 10. $P S_{1} \Rightarrow m F S$.

Proof. For every agent $i, p s_{1}(i)=\max _{\sigma \in \mathcal{A}^{\prime}} \max _{j \in N} u_{i}(\sigma(j))$ where $\mathcal{A}^{\prime} \subseteq$ $\mathcal{A}$ is the set of allocations giving to each agent exactly one object within $\left\{o_{i}^{k n+1}, o_{i}^{k n+2}, \ldots, o_{i}^{k n+n}\right\}$ for each $0 \leq k<m / n$. Thus, $p s_{1}(i) \geq \min _{\sigma \in \mathcal{A}^{\prime}}$ $\max _{j \in N} u_{i}(\sigma(j)) \geq \min _{\sigma \in \mathcal{A}} \max _{j \in N} u_{i}(\sigma(j))=m f s_{i}$. 
Nevertheless, $\mathrm{PS}_{1}$ is not stronger than EF since it does not even imply EF1.

Proposition 11. $P S_{1} \nRightarrow$ EF1, even under strict preferences over the objects.

Proof. Consider an instance where $n=4$ and $m=12$. The utilities are:

$$
\left(\begin{array}{cccccccccccc}
20 & 19 & 18 & 17 & 8 & 7 & 6 & 5 & 4 & 3 & 2 & 1 \\
15 & 12 & 11 & 10 & 6 & 3 & 9 & 5 & 13 & 8 & 7 & 2 \\
1 & 6 & 9 & 8 & 12 & 18 & 20 & 19 & 5 & 10 & 14 & 3 \\
2 & 5 & 6 & 15 & 11 & 12 & 9 & 8 & 4 & 20 & 18 & 19
\end{array}\right)
$$

Allocation $\sigma$ (circles), with $u(\sigma)=(32,33,57,57)$, is $\mathrm{PS}_{1}$ since $p s_{1}=(32,31,38$, 38 ). But agent 1 envies agent 2 , even if any object is removed from $\sigma(2)$.

The fact that $\mathrm{PS}_{1} \not \Rightarrow \mathrm{EF} 1$ may look surprising since fair guarantees of PS criteria can be interpreted via RB sequences, which generate EF1 allocations. However, satisfying a PS criterion does not impose to be the outcome of an RB sequence but focuses on the fulfillment of associated minimum utility levels, that are personal to each agent and do not need the inter-comparison between agents.

In their relaxed versions, $\mathrm{PS}_{1}$ and Prop remain connected.

Proposition 12. $P S_{1} c \Rightarrow$ Propc.

Proof. Take an instance, an agent $i$, and a $\mathrm{PS}_{1} c$ allocation $\sigma$. Recall that, w.l.o.g., $m=q n$. There exists $X^{i} \subseteq M \backslash \sigma(i)$ such that $\left|X^{i}\right| \leq c$ and $u_{i}(\sigma(i))+u_{i}\left(X^{i}\right) \geq$ $u_{i}\left(\left\{o_{i}^{1}, o_{i}^{1+n}, \ldots, o_{i}^{1+(q-1) n}\right\}\right)$. Since $u_{i}\left(\left\{o_{i}^{1}, o_{i}^{1+n}, \ldots, o_{i}^{1+(q-1) n}\right\}\right) \geq u_{i}(M) / n$, we get that $u_{i}(\sigma(i))+u_{i}\left(X^{i}\right) \geq u_{i}(M) / n$. Thus, $\sigma$ satisfies Prop $c$.

From Proposition 9, $\mathrm{PS}_{n}$ cannot imply Prop $c$ when the number of objects $m$ is large enough. However, $\mathrm{PS}_{n}$ implies Propc when $m$ is at most $(1+c) n$.

Proposition 13. If $m \leq(1+c) n$, then $P S_{n} \Rightarrow$ Propc.

Conversely, no "up to" relaxation of envy-freeness implies a PS criterion.

Proposition 14. EFX does not imply any PS criterion.

Proof. By Proposition 7 , we have that EFX $\nRightarrow \mathrm{PS}_{1} 1$. To prove that EFX $\nRightarrow$ $\mathrm{PS}_{n}$, consider an instance where $n=2$ and $m=4$. The utilities are:

$$
\left(\begin{array}{lllll}
12 & 8 & 12 & 8 \\
114 & 1 & 13 & 0
\end{array}\right)
$$

Allocation $\sigma$ (circles) is EFX: agent 2 is not envious and agent 1 is not envious if one good is removed from $\sigma(2)$. However, $\sigma$ is not $\mathrm{PS}_{n}$ since $p s_{n}=(20,13)$.

Proposition 2 states that EFX $\nRightarrow$ PropX though EF $c \Rightarrow$ Prop $c$. We know that $\mathrm{PS}_{1} 1 \Rightarrow$ Prop1 and it turns out that $\mathrm{PS}_{1} \mathrm{X} \Rightarrow$ PropX.

Proposition 15. $P S_{1} X \Rightarrow \operatorname{Prop} X$. 
Proof. Take an instance, an agent $i$, and a $\mathrm{PS}_{1} \mathrm{X}$ allocation $\sigma$. Recall that, w.l.o.g., $m=q n$. We have $u_{i}(\sigma(i))+u_{i}(x) \geq u_{i}\left(\left\{o_{i}^{1}, o_{i}^{1+n}, \ldots, o_{i}^{1+(q-1) n}\right\}\right)$ for all $x \in M \backslash \sigma(i)$. Since $u_{i}\left(\left\{o_{i}^{1}, o_{i}^{1+n}, \ldots, o_{i}^{1+(q-1) n}\right\}\right) \geq u_{i}(M) / n, u_{i}(\sigma(i))+u_{i}(x) \geq$ $u_{i}(M) / n$ holds for all $x \in M \backslash \sigma(i)$. Hence, $\sigma$ satisfies PropX.

$\mathrm{EFX} \Rightarrow \mathrm{PS}_{1} \mathrm{X}$ cannot hold because $\mathrm{EFX} \nRightarrow$ PropX and $\mathrm{PS}_{1} \mathrm{X} \Rightarrow$ PropX. Moreover, $\mathrm{PS}_{1} \mathrm{X} \Rightarrow \mathrm{EFX}$ cannot hold because it would contradict Proposition 11.

Note that $\mathrm{PS}_{n}$ gives a $\frac{1}{n}$-approximation for MMS, like EF1 [1]. The approximation ratio of a $\mathrm{PS}_{p}$ allocation can be generalized to $\frac{n-p+1}{n}$ for all $p \in[n]$.

Proposition 16. $\forall(p, i) \in[n] \times N, p s_{p}(i) \geq \frac{n-p+1}{n} m m s_{i}$.

\section{Efficiency of Fair Allocations}

It is long known that there is a tension between the two goals of computing efficient and fair allocations $[14,26]$. In this section we propose an empirical analysis of how the PS criteria go together with efficiency, and a comparison with the classical fairness criteria.

An allocation is Pareto-efficient if there is no other allocation $\sigma^{\prime}$ that Paretodominates it, i.e., such that $u_{i}\left(\sigma^{\prime}(i)\right) \geq u_{i}(\sigma(i))$ for each agent $i$, and the inequality is strict for at least one agent. The social welfare $(S W)$ is another efficiency measure. The utilitarian $S W$ of allocation $\sigma$ is equal to $\sum_{i \in N} u_{i}(\sigma(i))$, the egalitarian $S W$ to $\min _{i \in N} u_{i}(\sigma(i))$, and the Nash $S W$ to $\prod_{i \in N} u_{i}(\sigma(i))$.

It is easy to see that a Pareto-efficient allocation may not satisfy a PS criterion, and vice versa. Similarly, maximizing the social welfare and achieving PS fairness may be disconnected, as illustrated in the next example.

Example 2. Consider the instance given in the proof of Proposition 14. The allocation $\sigma$ (circles), which is the unique allocation maximizing the utilitarian, egalitarian and Nash SW, is not even $\mathrm{PS}_{n}$ since $u_{1}(\sigma(1))=16<20=p s_{n}(1)$. It is surprising since an allocation maximizing the Nash SW is known to be EF1 and to provide a good approximation to MMS [15]. Alternatively, the unique $\mathrm{PS}_{1}$ allocation (frames) is different and thus does not maximize any social welfare.

We study how often "fair" allocations are efficient. We run 1,000 instances with $n=3$ agents where $m$ ranges from 6 to 9 (adding dummy objects can make $m$ a multiple of $n$ ). The valuations of the agents over the objects are integers between 0 and 100 generated following a uniform distribution and then normalized. All the $n^{m}$ possible allocations are considered. We compare in Table 1 the percentage of allocations satisfying classical and PS fairness criteria, and the proportion of these allocations that are Pareto-efficient. Moreover, we compare in Fig. 2 the average of the utilitarian and egalitarian SW among the allocations satisfying a given fairness criterion (the behavior for Nash SW is similar).

In practice, the set of $\mathrm{PS}_{1} \mathrm{X}$ allocations is almost the same as the set of $\mathrm{PS}_{1}$ allocations, where $\mathrm{PS}_{1}$ is very demanding, even though less than CEEI. A significant proportion of allocations are $\mathrm{PS}_{n}(\approx 20 \%)$. This is less than the proportion 
Table 1. Percentage of allocations satisfying fairness criteria and percentage of these allocations that are Pareto-efficient for $n=3$

\begin{tabular}{ccccccccc}
\hline \multicolumn{4}{c}{ \% fair allocations } & \multicolumn{3}{c}{ \% Pareto-eff. alloc. / fair alloc. } \\
\hline$m$ & 6 & 7 & 8 & 9 & 6 & 7 & 8 & 9 \\
\hline MMS & 9.73 & 6.69 & 5.35 & 4.53 & 20.54 & 13.50 & 8.37 & 4.80 \\
Prop & 3.64 & 3.67 & 3.67 & 3.51 & 30.85 & 18.29 & 10.38 & 5.56 \\
mFS & 2.20 & 2.60 & 2.94 & 3.06 & 38.00 & 21.77 & 11.73 & 6.05 \\
EF & 0.83 & 0.86 & 0.82 & 0.77 & 51.15 & 32.44 & 20.22 & 11.67 \\
CEEI & 0.39 & 0.25 & 0.13 & 0.07 & 86.31 & 80.34 & 80.21 & 82.28 \\
\hline Prop1 & 79.28 & 72.99 & 68.50 & 64.49 & 7.94 & 4.43 & 2.33 & 1.19 \\
PropX & 4.87 & 4.5 & 4.37 & 4.13 & 23.02 & 14.72 & 8.58 & 4.71 \\
\hline EF1 & 18.65 & 17.04 & 15.42 & 14.21 & 13.65 & 7.90 & 4.30 & 2.22 \\
EFX & 2.85 & 2.33 & 1.88 & 1.66 & 29.03 & 18.85 & 12.25 & 7.11 \\
\hline $\mathrm{PS}_{3}$ & 30.69 & 18.43 & 17.46 & 18.94 & 11.77 & 8.03 & 4.31 & 2.12 \\
$\mathrm{PS}_{2}$ & 4.01 & 4.27 & 5.42 & 3.73 & 28.38 & 16.76 & 8.25 & 5.61 \\
$\mathrm{PS}_{1}$ & 0.15 & 0.43 & 0.22 & 0.26 & 83.67 & 50.26 & 44.63 & 26.02 \\
\hline $\mathrm{PS}_{1} 1$ & 46.76 & 49.53 & 36.48 & 34.60 & 9.16 & 5.13 & 2.97 & 1.51 \\
$\mathrm{PS}_{1} \mathrm{X}$ & 0.16 & 0.50 & 0.26 & 0.30 & 82.36 & 41.72 & 33.52 & 22.14 \\
\hline
\end{tabular}

of $\mathrm{PS}_{1} 1$ allocations $(\approx 40 \%)$, and even significantly less than the proportion of Prop1 allocations $(\approx 70 \%)$. Among these 3 criteria which can be satisfied for any instance, our experiments show that $\mathrm{PS}_{n}$ is the most selective one.

We observe that the proportion of efficient allocations among fair allocations seems to be dependent on the number of fair allocations. In particular, the proportion of $\mathrm{PS}_{1}$ allocations that are Pareto-efficient is superior to the same quantity for $\mathrm{EF}$ allocations, and the utilitarian $\mathrm{SW}$ for $\mathrm{PS}_{1}$ is also better in average than the SW for EF. EF1 and $\mathrm{PS}_{n}$, which are both always satisfiable, seem to be equivalent regarding efficiency.

\section{Conclusion}

We have introduced some criteria whose fair guarantees are inspired by picking sequences where every agent supposes that her turns are the multiples of $p$. By the simplicity of the picking sequence protocol and the definition of the PS fair guarantee, PS criteria can be easily expressed by an agent as a fairness requirement. Moreover, even without knowing how the rest of goods is allocated, it is easy for an agent to check whether she can be satisfied with her own assigned bundle according to a PS criterion. The two extreme criteria $\left(\mathrm{PS}_{1}\right.$ and $\left.\mathrm{PS}_{n}\right)$ are well connected with the ordered scale of fairness. More precisely, $\mathrm{PS}_{1}$ implies mFS, MMS implies $\mathrm{PS}_{n}$, whereas $\mathrm{PS}_{1} 1$ and $\mathrm{PS}_{1} \mathrm{X}$ imply Prop1 and PropX, respectively. In light of the fact that EFX $\nRightarrow$ PropX, the connection of relaxed PS1 with relaxed proportionality is interesting and highlights that PS criteria and proportionality are conceptually close. We have proved that allocations satisfying $\mathrm{PS}_{n}$ and $\mathrm{PS}_{1} 1$ can always be found whereas, as showed via our extensive comparative study of PS criteria with classical fairness criteria, these two criteria are far from being trivial. This positive result regarding the possibility of satisfaction is appealing since only a few known fairness criteria (EF1 and Prop1 


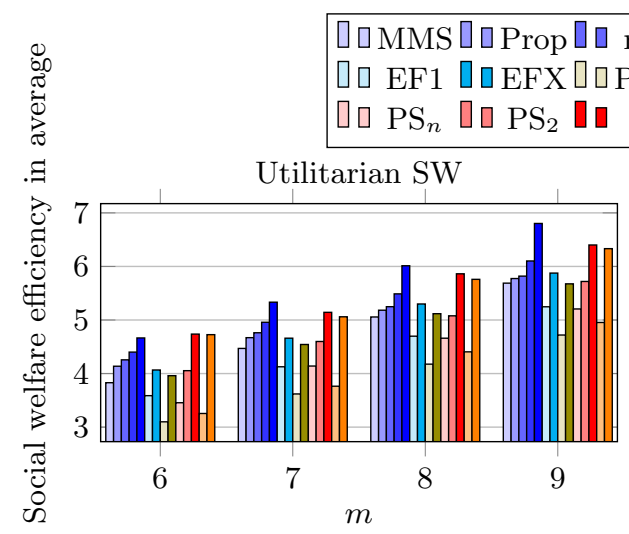

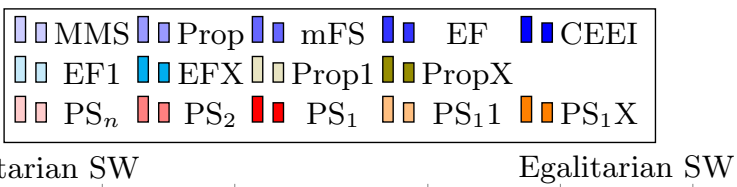

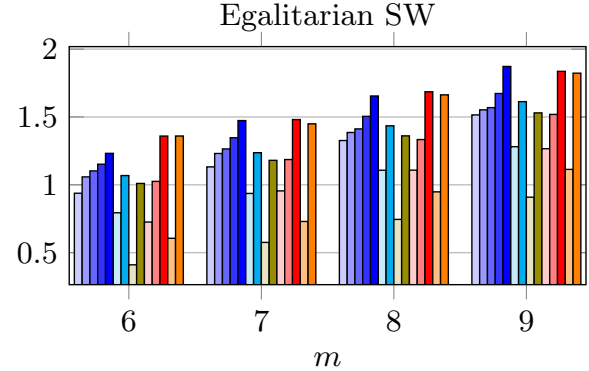

Fig. 2. Average of the utilitarian and egalitarian social welfares within the set of fair allocations for $n=3$ and $m \in\{6,7,8,9\}$

among those studied here) are always satisfiable. The whole picture of existence and interactions between fairness criteria is depicted in Figure 1. Combined with the experiments which explore the compatibility of the PS criteria with efficiency, our work contributes to the understanding of how the fairness criteria for allocating indivisible goods interact.

The fact that EFX $\nRightarrow$ PropX when $n>2$ (Proposition 2) calls for the following less restrictive notion of envy-freeness: Allocation $\sigma$ is broadly $\mathrm{EF} c$ (bEFc) iff for every pair of agents $i$ and $j$, there exists $X^{i} \subseteq M \backslash \sigma(i)$ such that $\left|X^{i}\right| \leq c$ and $u_{i}(\sigma(i))+u_{i}\left(X^{i}\right) \geq u_{i}(\sigma(j))$. Allocation $\sigma$ is broadly EFX (bEFX) iff for every pair of agents $i$ and $j, u_{i}(\sigma(i))+u_{i}(x) \geq u_{i}(\sigma(j))$ holds for any $x \in M \backslash \sigma(i)$. Then, $\mathrm{EF} c \Rightarrow \mathrm{bEF} c \Rightarrow \operatorname{Prop} c$, $\mathrm{bEFX} \Rightarrow \mathrm{EFX}$, and $\mathrm{bEFX} \Rightarrow$ PropX hold. Obviously, EFX and bEFX coincide when $n=2$.

Several research directions can be derived from this work. Alternative definitions of fair guarantees can be explored like what some agent $i$ would get in a picking sequence with a balanced alternation policy $\langle 1, \ldots, n|n, \ldots, 1| \ldots\rangle$. Another possibility would be to consider a probability distribution over the possible positions taken by the agent in round robin. By using a uniform distribution, we fall back to the definition of proportionality. One can also suppose that $p$ in $\mathrm{PS}_{p}$ is not the same for all agents.

We have focused on a particular type of relaxation of a fairness criterion $F$, namely when $F$ can be satisfied up to some good(s). Other relaxations can be studied. For example, $F$ can be satisfied for any given subset of $t$ privileged agents. Another type of relaxation consists of satisfying $F$ up to a multiplicative factor. We know that $\mathrm{PS}_{n}$ can always be satisfied (Proposition 5) but when $p<n$, no positive $\alpha$ guarantees the existence of an allocation $\sigma$ such that $u_{i}(\sigma(i)) \geq \alpha p s_{p}(i)$ for all $i \in N$. 


\section{Bibliography}

[1] Amanatidis, G., Birmpas, G., Markakis, E.: Comparing approximate relaxations of envy-freeness. In: Proc. of IJCAI'18. pp. 42-48 (2018)

[2] Aziz, H., Bouveret, S., Lang, J., Mackenzie, S.: Complexity of manipulating sequential allocation. In: Proc. of AAAI'17. pp. 328-334 (2017)

[3] Aziz, H., Huang, X., Mattei, N., Segal-Halevi, E.: The constrained round robin algorithm for fair and efficient allocation. arXiv preprint arXiv:1908.00161 (2019)

[4] Aziz, H., Walsh, T., Xia, L.: Possible and necessary allocations via sequential mechanisms. In: Proc. of IJCAI'15. pp. 468-474 (2015)

[5] Bogomolnaia, A., Moulin, H., Stong, R.: Guarantees in fair division: general or monotone preferences. arXiv preprint arXiv:1911.10009 (2019)

[6] Bouveret, S., Chevaleyre, Y., Maudet, N.: Fair allocation of indivisible goods. In: Handbook of Computational Social Choice, chap. 12, pp. 284310. Cambridge University Press (2016)

[7] Bouveret, S., Lang, J.: A general elicitation-free protocol for allocating indivisible goods. In: Proc. of IJCAI'11. pp. 73-78 (2011)

[8] Bouveret, S., Lang, J.: Manipulating picking sequences. In: Proc. of ECAI'14. pp. 141-146 (2014)

[9] Bouveret, S., Lemaître, M.: Characterizing conflicts in fair division of indivisible goods using a scale of criteria. Auton. Agents Multi-Agent Syst. 30(2), 259-290 (2016)

[10] Brams, S.J., Edelman, P.H., Fishburn, P.C.: Fair division of indivisible items. Theory and Decision 55(2), 147-180 (2003)

[11] Brams, S.J., King, D.L.: Efficient fair division: Help the worst off or avoid envy? Rationality and Society 17(4), 387-421 (2005)

[12] Brams, S.J., Taylor, A.D.: The win-win solution: guaranteeing fair shares to everybody. WW Norton \& Company (2000)

[13] Budish, E.: The combinatorial assignment problem: Approximate competitive equilibrium from equal incomes. Journal of Political Economy 119(6), 1061-1103 (2011)

[14] Caragiannis, I., Kaklamanis, C., Kanellopoulos, P., Kyropoulou, M.: The efficiency of fair division. Theory Comput. Syst. 50(4), 589-610 (2012)

[15] Caragiannis, I., Kurokawa, D., Moulin, H., Procaccia, A.D., Shah, N., Wang, J.: The unreasonable fairness of maximum Nash welfare. ACM Trans. Economics and Comput. 7(3), 12:1-12:32 (2019)

[16] Chakraborty, M., Igarashi, A., Suksompong, W., Zick, Y.: Weighted envyfreeness in indivisible item allocation. In: Proc. of AAMAS'20. pp. 231-239 (2020)

[17] Conitzer, V., Freeman, R., Shah, N.: Fair public decision making. In: Proc. of EC'17. pp. 629-646 (2017)

[18] Foley, D.K.: Resource allocation and the public sector. Yale Economic Essays $7(1), 45-98$ (1967) 
[19] Kalinowski, T., Narodytska, N., Walsh, T., Xia, L.: Strategic behavior when allocating indivisible goods sequentially. In: Proc. of AAAI'13. pp. 452-458 (2013)

[20] Kohler, D.A., Chandrasekaran, R.: A class of sequential games. Operations Research 19(2), 270-277 (1971)

[21] Kurokawa, D., Procaccia, A.D., Wang, J.: When can the maximin share guarantee be guaranteed? In: Proc. of AAAI'16. pp. 523-529 (2016)

[22] Lang, J., Rothe, J.: Fair division of indivisible goods. In: Rothe, J. (ed.) Economics and Computation: : An Introduction to Algorithmic Game Theory, Computational Social Choice, and Fair Division, chap. 8, pp. 493-550. Springer (2016)

[23] Lipton, R.J., Markakis, E., Mossel, E., Saberi, A.: On approximately fair allocations of indivisible goods. In: Proc. of EC'04. pp. 125-131 (2004)

[24] Moulin, H.: Cooperative Microeconomics: A Game-Theoretic Introduction. Princeton University Press (1995)

[25] Moulin, H.: Fair Division in the Internet Age. Annual Review of Economics 11(1), 407-441 (2019)

[26] Procaccia, A.D.: Cake cutting algorithms. In: Handbook of Computational Social Choice, pp. 311-330. Cambridge University Press (2016)

[27] Procaccia, A.D., Wang, J.: Fair enough: Guaranteeing approximate maximin shares. In: Proc. of EC'14. pp. 675-692 (2014)

[28] Steinhaus, H.: The problem of fair division. Econometrica 16(1), 101-104 (1948)

[29] Tominaga, Y., Todo, T., Yokoo, M.: Manipulations in two-agent sequential allocation with random sequences. In: Proc. of AAMAS'16. pp. 141-149 (2016)

[30] Varian, H.R.: Equity, envy, and efficiency. Journal of Economic Theory 9(1), 63-91 (1974) 